\title{
9 Systemic analysis of the nexus of greenhouse gas emissions and material use in the energy sector
}

\author{
Ullrich Lorenz
}

\section{The starting point}

The combustion of fossil fuels is one of the major sources of greenhouse gas (GHG) emissions and a primary cause of climate change. One focus of climate policy is to address this issue by demanding the transition from fossil-based energy production towards renewable energy. Such a transition refers to all areas where fossil fuels are currently used, both in industrial production and the private sector, including electricity generation, heating and transportation.

On the other hand, resource efficiency policy seeks to minimise the use of resources by increasing the efficiency of material use (providing the same service with less material), substituting (using substances less harmful to the environment) and simply abstaining from material use (sufficiency).

Material use and energy use are inherently interconnected. All steps in the value chain require energy - the extraction of raw materials, the preparation, transportation, and production of products, and the consumption, use and finally the deposition or recycling of the materials. Additionally, the energy system heavily relies on raw materials, namely fossil fuels like lignite, coal, oil and gas. It makes a huge difference if the material is burned, i.e. chemically and physically altered (with increased entropy), or just transformed into metals which remain metal and can be recycled. Although entropy will also be increased when these metals are distributed in fine particles that cannot be recaptured and recycled and are also lost for further use.

In order to substitute fossil fuels in the energy system, the clear objective should be the replacing of fossil power stations with renewable energy systems. Whatever renewable energy system will is built (e.g. wind power, solar, thermal), materials like concrete, steel, copper, silver, aluminium and other raw earth materials needed for electronics will be used. However, each kilowatt-hour that is delivered by renewable energy systems could theoretically save fossil fuels, assuming the energy demand remains the same and fossil production is reduced at the same rate. Greenhouse gas emissions associated with investments in materials for the renewable energy systems have to be balanced with a reduction in fossil fuel combustion. This way, instead of continuously reducing the stock of fossil fuels, the material is accumulated in 
power units and builds up an anthropogenic stock of - at least theoretically reusable materials.

Hence, the area of climate policy that fosters the transition to a renewable energy system has a direct impact on material use and vice versa. On the other hand, a policy that focuses only on the efficiency of raw material use alone has potential to influence $G H G$ emissions for the good and the bad, although currently this potential is not used in a systematic way for the good.

\section{Systemic approaches}

\section{System analysis}

The word 'system' comes from Greek and means 'a regularly interacting or interdependent group of items forming a unified whole' (Webster's 2019). Systems science is referred to here as the science of systems thinking, systems analysis and systems dynamics. Understanding the connections and relations of the different parts of the system is necessary to understand a certain behaviour of the system (Haraldsson 2004). Understanding a cause and an effect enables the analysis, determination and explanation of how changes come about under certain conditions (Dörner 1996).

This approach also requires one defining the system boundaries. This means the research question defines the extent of the system, which is at first the mental model and collection of connected factors that define and help to explain a certain behaviour. Human thinking tends to be generally linear, not taking into account feedbacks, especially in politics. While in many situations, linear thinking is effective, in complex context linear thinking has its limits (Haraldsson 2004).

There are certain characteristics that appear in the behaviour of systems. Feedback loops can either lead to reinforcing exponential growth (positive or negative) or to a balancing structure (goal-seeking). In complex system ambiguities appear, which means that one factor can affect another factor through different causal pathways - sometimes in opposite that is neutralising directions. Another common effect in systems are delays, where a change in one factor does not immediately change another factor but instead, it takes some time before the effect manifests. A combination of such delays can often lead to a form of oscillatory behaviour in systems. Considering these combined processes, human brains are not able to explain such complex behaviours of a system without the help of tools and a methodology to apply them. Additionally, when a system is a network of multiple variables that are connected to each other through causal relationships and their combination expresses some behaviour, this can only be characterised through observation of the whole system (Sterman 2000). Qualitative modelling is one of the approaches to understand and analyse complex systems. 


\section{Qualitative modelling}

Qualitative modelling is a form of structural modelling in which gaining an approximate understanding of the (causal) relations and interconnections of factors and concepts is the central aim (Haraldsson 2004). In quantitative modelling, in addition, the objective is to simulate concrete figures for a certain point of time (Lendaris 1980). This article focusses on the result of a purely qualitative exercise. Regardless of the tool used, it is always essential that decisive factors and their relations, are included in the model.

In this chapter, a causal loop diagram (CLD), as known from system dynamics (e.g. Sterman 2000), is presented, analysed and discussed. When connecting two or more factors in a qualitative model, additional information is added in relation to the causal effect. These include the direction of the effect, whether the causal effect is in the same direction (more causes more/less causes less) or opposite direction (more causes less/less causes more), its strength/weighting, and any delay it may involve (e.g. Neumann 2013). Using knowledge of the dominant loops and loop structure, qualitative modelling allows for rough estimates about how a system could behave.

\section{The nexus of greenhouse gas emissions and material use}

\section{Understanding the system}

The nexus between greenhouse gas emissions and material use in the energy sector can be modelled and displayed using a CLD as shown in Figure 9.1. Both Production and Consumption require energy in our society. More Production leads to more Consumption (import/export equals consumption/ production somewhere else) and vice versa. If considered by itself, this reinforcing feedback loop would lead to an exponential growth of Production and Consumption (and therefore to an exponential growth of energy use and of the use of raw materials). Theoretically, Production would be limited by the Available material, although under current conditions this limitation is physically not given. Also, either fossil or renewably produced energy that is available for use could be a limitation to the production processes, although this is currently not the case.

Actual consumption has grown over recent years without a limitation of the (geo)physical system manifesting and constraining growth, though this may change. One has to acknowledge that the economic system as a whole is not shown here. Hence, economic factors like price and available money, purchasing power, among others which balance out the production/consumption loop, have been omitted in the model in Figure 9.1. These factors might dump or slow down the dynamics of production and consumption. Nevertheless, for the generic understanding of the nexus these details are not essential at this level of analysis.

Energy (heat, kinetic or electric power) - represented as Energy demand in the CLD could either be produced by fossil energy (Fossil energy production) 


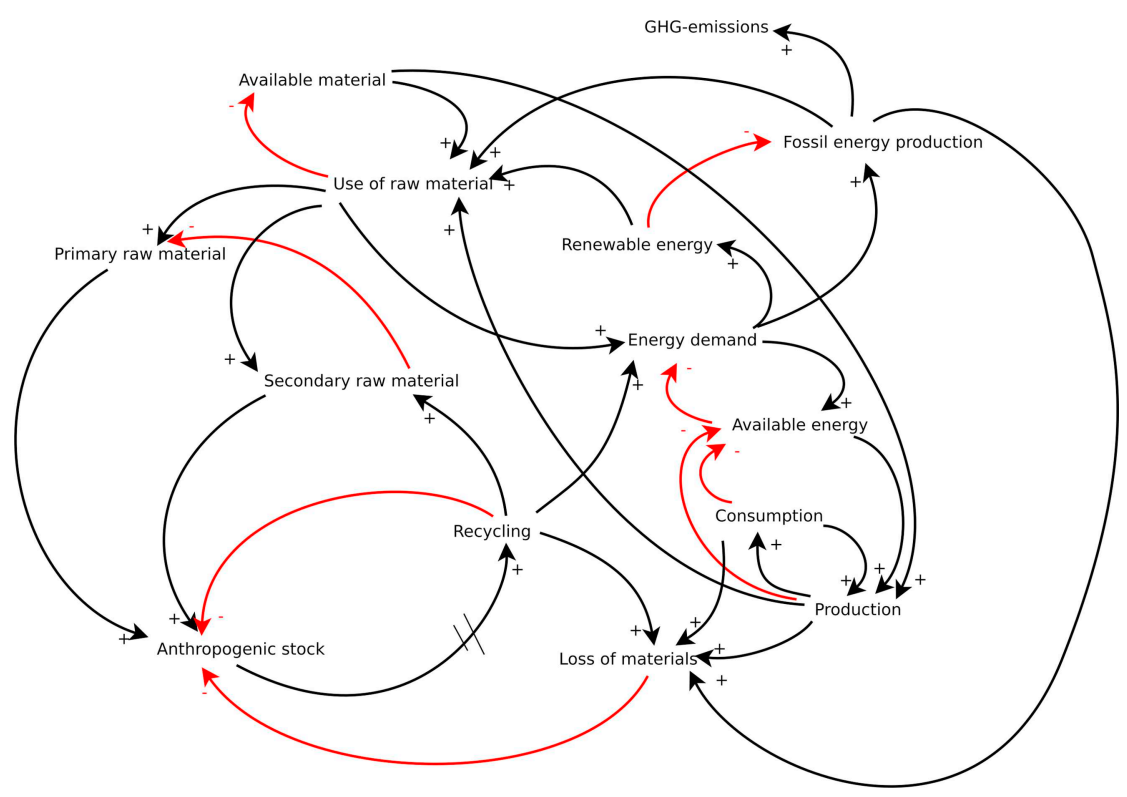

Figure 9.1 Causal loop diagram (CLD) of the nexus of greenhouse gas emissions and material use in the energy sector. Arrows with a '+' (black) represent an equal causal relation (more leads to more or less leads to less), while arrows with a '-' (red) represent an opposing relation (more leads to less or less leads to more). Source: own compilation, first used in UBA (2019).

or renewably (Renewable energy). The 'burning' of fossil fuels requires raw materials. The same is true for the construction of infrastructure to produce renewable energy which also requires raw materials (e.g. steel needs iron-ore and fossil fuel for smelting). As mentioned in the introduction, there is a key difference between the Use of raw material due to the burning of fossil fuels which are lost after burning (the connection between Fossil energy production and Loss of materials)and the building of Renewable energy production units. The units there add to the Anthropogenic stock and could be recycled later.

The Use of raw materials depends on Available material. Additionally, the Use of raw materials will be covered either by Primary raw material, which includes extraction and transport of raw material, or the demand will be fulfilled by Secondary raw material which comes from Recycling. In any case, the use of secondary materials and the use of primary materials comprise the Anthropogenic stock of materials. The more we use Secondary raw material, the less we need Primary raw material extraction. While Recycling is temporarily taking away material from the Anthropogenic stock, it is refilled by Secondary materials. The only process truly diminishing the Anthropogenic stock is the Loss of materials. This could be either dispersion of materials, chemical transformation such as burning and the final deposition of ashes, the contamination of material which 
might lead to unrecycled and hence deposited materials. Thus, the Loss of materials is a result of losses during the production process, during Consumption, during Recycling - with current recycling rates well below 100\% - and during Fossil energy production.

\section{Understanding the central loops}

The CLD shown in Figure 9.1 contains 32 loops, where 15 loops are balancing and 17 loops are reinforcing. The loops are feedback structures, where a cause becomes an effect and vice versa. Following Senge (1990), no one factor is responsible for changes in the whole system. Therefore it is essential to understand loop interactions and a possible resulting behaviour in order to understand the system and the impacts of action and inaction. This knowledge can help policy analysts identify where the system measures are needed and how to design them leveraging the existing loops. Basically, a reinforcing loop creates either exponential growth while a balancing loop will show goal-seeking behaviour. Certain structures like delays in loop structures (e.g. reinforcing together with balancing loops) normally create some oscillation in the system (Haraldsson 2004). Mostly we find combinations of loops in systems. Certain combinations can create specific patterns which are called systems' archetypes. A very common combination is the connection of a reinforcing and a balancing feedback loop, which in isolation creates a logistic growth function (exponential growth which is slowed down and ends goal-seeking). In combination with a delay an overshot and collapse could be the result (Senge 1990; Lorenz et al. 2017).

\section{The anthropogenic stock and recycling}

One loop combination is formed around the anthropogenic stock. The anthropogenic material stock consists of the accumulated materials in buildings, infrastructure and durable goods. This stock constitutes a valuable reservoir of secondary raw materials (Schiller et al. 2017). The anthropogenic stock is contributed to either by primary raw materials or secondary raw materials (Figure 9.2). The process of recycling feeds back across secondary materials, despite a certain loss of material and consideration of energy costs. The linear flow from primary raw material to the anthropogenic stock has led - and is still leading - to the accumulation of material we are experiencing currently. Considering the reinforcing loop from Anthropogenic stockRecycling - Secondary raw material, theoretically the stock would remain stable if not for the delay and the losses due to recycling. This delay and the recycling may lead to oscillations and decay around the anthropogenic stock. The secondary raw materials are not an immediate available.

This has consequences for policy design. Before one could think of using notable amounts of material from the anthropogenic stock, this stock must contain sufficient material to be recycled. If a policy, for example, creates a 


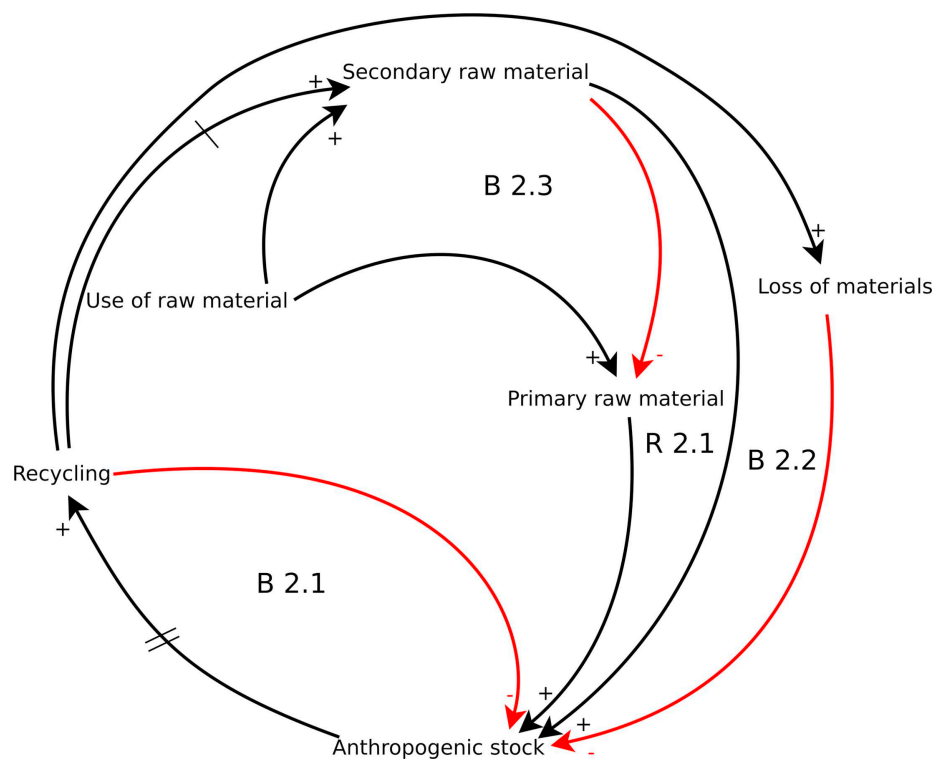

Figure 9.2 Excerpt from the CLD in figure 9.1, showing the central loops around the anthropogenic stock. B 2.1 shows a balancing loop which might reduce the anthropogenic stock; B2.2 marks the balancing loop that through recycling also material is lost. B2.3 shows the balancing of the primary raw material input to the anthropogenic stock. R 2.1 refers to the reinforcing loop filling up the anthropogenic stock.

Source: own compilation.

reduction in the use of primary raw materials (e.g. by the application of taxes or restrictions of exploration/exploitation), it is likely to lead to shortages in production. It will take some time until secondary materials become available and are used. Importantly, this would require installing the necessary additional recycling capacities. Even when recycling processes are fully established, the losses inherent to recycling require a constant inflow of primary raw materials if society wants to keep anthropogenic stock constant. In any case, policy approaches to minimise losses of materials and increase recycling capacities are necessary amendments.

\section{The nexus of energy and resource use}

Another crucial combination of loops is centred on the nexus between GHG emissions and the use of raw material (see Figure 9.3). The central 'engine' for the production of GHG emissions is the reinforcing loop $\mathrm{R}-3.1$ in the figure: more energy demand leads to more fossil power production, which requires fossil raw materials. This flow creates a double adverse effect: next to greenhouse 


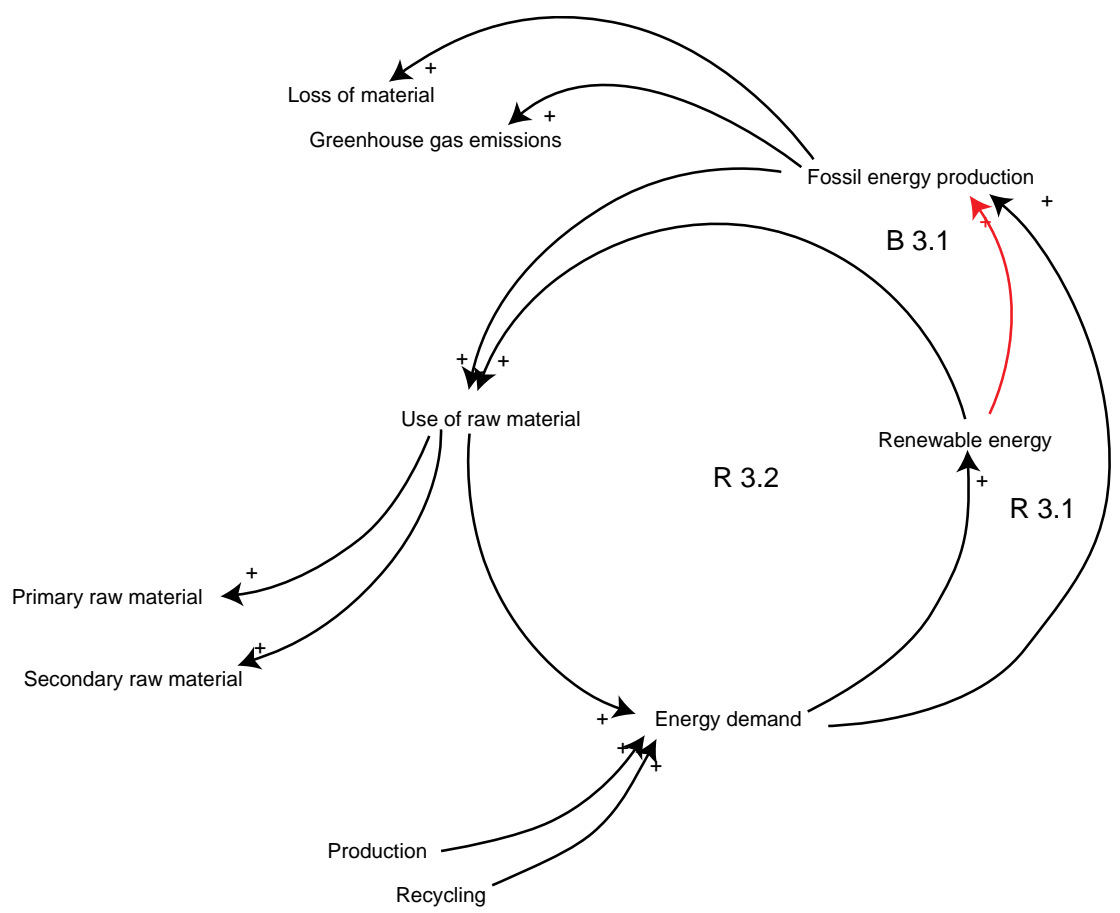

Figure 9.3 Excerpt from the CLD showing the central loops around the nexus of the use of raw material and Greenhouse gas emissions. R3.1 marks the reinforcing loop that more fossil energy production requires more raw material which require more energy. R 3.2 refers to the reinforcing loop that more renewable energy requires more use of raw material. The balancing loop B 3.1 shows the possible reduction of fossil energy production due to increase of renewable energy sources.

Source: own compilation.

gas emissions, the fossil material is lost for further use. The use of raw material includes exploitation and transportation, requiring energy as an investment (see the article of Sverdrup et al.). The same loop is active when accounts for this. At the same time, renewable energy infrastructure may be built $(\mathrm{R}-3.2)$, with the difference that this does not lead to material loss like when burning fossil fuels. The balancing loop B -3.1 is responsible for the reduction of GHG emissions.

Another pathway to reduce GHG emissions could be the direct reduction of energy demand. However, energy demand (and thus any reduction thereof) is mainly driven by the provision of raw materials, production and consumption. As long as production and consumption rates are high, the only efficiency can reduce the demand for energy. As long as renewable energy infrastructure is built, the use of raw material and energy demand will stay high or even increase, even if demand from fossil fuel use is already on the decline. It must be clear to policy and society that the shift to a renewable energy system itself 
will cost energy, raw materials and time. As long as energy demand is met by an energy mix that contains fossil fuels, the construction of renewable energy infrastructure creates additional emissions of GHG. On the other hand, each renewable energy site contributes to the longer-term reduction of GHG emissions. Additionally, the construction of renewable energy sites and the production of renewable energy create regional welfare and attract (further) investment. One preliminary conclusion for policy is the need to take the amount of time needed for effects to be manifested into consideration and that certain measures require investments, a return on which will take time.

\section{The consumption and production loop}

People are consuming things. Some of these fulfil basic needs like nutrition, housing, communication or mobility. All such things (including the required infrastructure) have to be produced. The economy is centred around this loop of supply and demand - the more that is demanded, the more will be produced, and the more that is produced, the more will be consumed, and so on. Both processes, production and consumption, require energy and are leading to loss of materials. The production loop $(\mathrm{R}-4.1$ in Figure 9.4) is also driving the use of raw material and the balancing loops $(\mathrm{B}-4.2$ and $\mathrm{B}-4.1)$ are limiting (theoretically) the production. Currently, most raw materials are abundant (although global imbalances and inequities are prevalent). However, the more effort it takes to provide raw materials, the more expensive provision gets and the more critical these raw materials might be from an economic perspective. This economic perspective is not included in this CLD. Nevertheless, the connection of loops shown in Figure 9.4 is a prototype of the limits to growth archetype, showing either a logistic growth curve which eventually stabilises on a high level or more likely leads to some overshot and collapse behaviour (Senge 1990). This prototype is already discussed in Lorenz et al. (2017) with respect to certain megatrends.

\section{Discussion and consequences for policy design}

The knowledge of the central feedback loops and causal connections in the nexus-system helps to understand the system's development tendencies and thus to design better policy approaches. It is very likely that sectoral policy will fail since it only addresses certain areas in the system and feedback loops might counteract or partially overcompensate its intention. Another common effect is that a certain measure might act on the central problem but does not resolve it or may in fact cause other problems (shifting the burden). In a good governance system, decisions should be taken on a well-informed basis, finding a systemic solution. In the case of the nexus between GHG emissions and the use of raw materials, it would not be a systemic solution to either only optimise the material use system or to only minimise the GHG at any cost. If the sole focus was given to the renewable energy systems, of course, the use of 


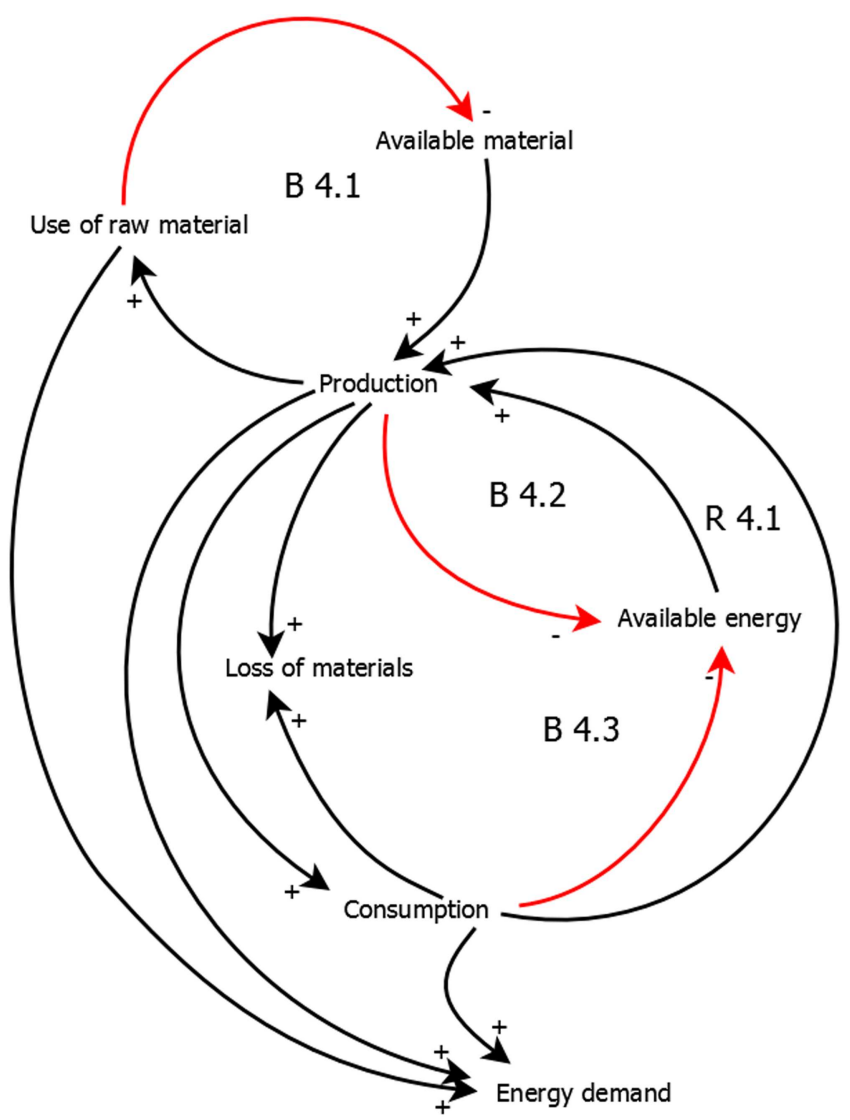

Figure 9.4 Excerpt from the CLD showing the central loops around production and consumption. B 4.1 marks the balancing loop showing the dependence between production of the raw material. B 4.2 shows the limitation of production from the available energy. The loop B 4.3 marks the balancing effect of consumption requiring energy which might (theoretically) limit production. The reinforcing loop R 4.1 shows the production-consumption 'engine'.

Source: own compilation.

fossil fuels (and their GHG emissions) would be reduced. On the other hand, raw materials like copper, aluminium, lithium, and rare earth metals are essential for this, thereby shifting the burden to an extent. The extraction and transport of raw materials cost energy. If other levers in the system are not pulled simultaneously, adverse short-term effects occur. Popular sentiments in German media these days criticise E-mobility or the Energy Transition ('Energiewende') with exactly these 'sectoral' arguments - that more resources and more energy is needed and that this would increase costs and have adverse economic effects and will produce more GHG. 
The systemic view, however, suggests a different view: parallel scaling up of recycling capacities, increasing the energy and material efficiency in production, reducing consumption levels (this relates to reduce, reuse, repair, recycle concepts) and closing the secondary loop (minimising material losses at all stages). Of course, most of these concepts and ideas are already known. Interestingly, however, they are promoted by different actors focusing on induvial aspects of the issue and are not brought together into a coherent movement and therefore action is lacking. For example, if focus would only be given to consumption, the effect on GHG emissions could be significant but might be offset by motivations of various actors. The interest of the economy is in this case to produce and to sell. As long as consumers, producers and policy makers are not moving in the same direction they will counteract each other. If policy actors embraced 'consequent' decisions, they (or their affiliates) would be punished at the next election. If a company would decide to produce less and/or only sustainable goods without demand, it would disappear from the market. What motivation does the fossil fuel industry have to stop their business when there are no alternatives? There is no motivation for a consumer who made an expensive investment in a car not use it anymore. The interests of different actors are blocking decisions and progress. This effect is described as a lock-in effect of actors (by Daschkeit et al. 2014).

To overcome this effect, this systemic and a more generalised perspective is helpful. The whole system will only 'move' in the right direction when several levers are pulled at the same time. Public investments are necessary, and revenue will emerge after a certain amount of time. Supporting a common (and well-informed) vision and with this, shifting common value settings will be the result which enables more sustainable consumer decisions. Thus it is the role of (eventually) international policy to change the framework conditions so that industry has incentives to transform and consumers are incentivised to change their consumption patterns based on common values.

\section{List of abbreviations}

B balancing loop

CLD causal loop diagram

GHG greenhouse gas

$\mathrm{R}$ reinforcing loop

\section{References}

Daschkeit, A., Kristof, K., Lorenz, U., and Veenhoff, S. (2014). Deutschland 2050. Bausteine für eine nachhaltige Zukunft in Jahrbuch Ökologie.

Dörner, D. (1996). The Logic of Failure, Recognizing and Avoiding Error in Complex Situations. Perseus Books, Reading.

Haraldsson, H. (2004). Introduction to System Thinking and CLD. Web version. Department of Chemical Engineering, Lund University. 
Lendaris, G. G. (1980). Structural Modeling: A Tutorial Guide. IEEE Transactions on Systems, Man and Cybernetics 10(12): 807-840.

Lorenz, U., Sverdrup, H. U., and Ragnarsdottir, K. V. (2017). Global Megatrends and Resource Use - A Systemic Reflection. In H. Lehmann (ed.), Factor X: Challenges, Implementation Strategies and Examples for a Sustainable Use of Natural Resources, 67-77. Springer Verlag, Frankfurt.

Neuman, K. (2013). 'Know Why' Thinking as a New Approach to Systems Thinking. E:CO Issue 15(3): 81-93.

Schiller, G., Müller, F., and Ortlepp, R. (2017). Mapping the Anthropogenic Stock in Germany: Metabolic Evidence for a Circular Economy. Resources, Conservation and Recycling 123: 93-107. 10.1016/j.resconrec.2016.08.007.

Senge, P. (1990). The Fifth Discipline. The Art and Practice of the Learning Organisation. Century Business, New York.

Sterman, J. D. (2000). Business Dynamics, System Thinking and Modelling for a Complex World. Irwin McGraw-Hill, New York.

UBA (2019). A Resource Efficient Pathway towards a Greenhouse Gas Neutral Germany. 2nd edition. Retrieved from www.umweltbundesamt.de/sites/default/files/medien/376/ publikationen/190212_uba_fachbrosch_rtd_engl_bf_low2.pdf.

Webster's. (2019). System. Retrieved from www.merriam-webster.com/dictionary/system visited September 2019. 